\title{
Simulation study of micelle formation by bile salts
}

\author{
Ana Vila Verde ${ }^{1}$ and Daan Frenkel ${ }^{2}$ \\ ${ }^{1}$ FOM institute AMOLF, Science Park 102, 1098 XG Amsterdam, The Netherlands, \\ a.vilaverde@amolf.nl \\ ${ }^{2}$ University of Cambridge, Lensfield Road, Cambridge CB2 1EW, UK, \\ df246@cam.ac.uk
}

February 15, 2010

\begin{abstract}
We report coarse-grained, implicit-solvent simulations of aqueous solutions of bile salts. The parameters in our model were optimized to reproduce some of the experimentally known behavior of dihydroxy bile salts at "physiological" temperature and counterion concentration. We find that micelle formation in dihydroxy and trihydroxy bile salts is only weakly cooperative in the sense that there is barely a free energy barrier that stabilizes these micelles against disassembly. Bile molecules are found to pack in many different orientations in pure bile micelles. Both features may be physiologically relevant: the ability to pack in different orientations may be necessary to form mixed micelles with nutrients of a wide range of molecular lengths and shapes, and the reduced micelle stability may facilitate nutrient release once the mixed micelles reach the intestinal wall.
\end{abstract}

\section{Introduction}

Bile salts and bile acids are surfactants that are important for the digestion of fats and fat-soluble nutrients by humans: the rate and extent to which nutrients are solubilized in dietary mixed micelles (DMMs, composed of bile salts, phospholipids, cholesterol and solubilized nutrients) are directly related to the rate and extent of absorption by intestinal cells ${ }^{1,2}$. A good understanding of the relation between DMM composition and uptake by the body is important because of its potential relevance for improving health and nutrition. By controlling the size and shape of particles and droplets of different nutrients/drugs in processed 
food products or medications, the uptake of particular nutrients or drugs into the body may be tuned. At present this can only be done empirically because the molecular-scale mechanisms by which nutrients are taken up in bile micelles and subsequently released near intestinal cells are poorly understood. Understanding these mechanisms requires molecular-scale models of the various components of DMMs as well as good understanding of the molecular scale behavior of DMM constituents. Surprisingly, bile salts are among the least studied at the molecular scale of all DMM components. Yet they play a crucial role in food uptake; the present paper focuses on their properties.

Bile salts and acids differ from the other amphiphiles in DMMs (phospholipids, fatty acids, monoglycerides) and from better-studied, head-tail surfactants such as sodium-dodecyl sulfate both in their molecular structure and in their surfactant properties. The basic structure of all bile salts (BS) consists of a rigid steroid backbone with a hydrophobic and a hydrophilic face to which a short and flexible tail is attached. Several different bile salts are present in the human body ${ }^{1,2}$. They may be conjugated with glycine or taurine molecules, thus becoming more soluble, or exist in unconjugated form. Bile salts may also differ in the number of hydroxyls (two or three) attached to the steroid group, the trihydroxy variety being more soluble. Different bile salts will nevertheless behave in a qualitatively similar manner: at physiological $\mathrm{NaCl}$ concentration $(0.15 \mathrm{M})$ and temperature, pure bile salts have high critical micellar concentrations (CMC), on the order of 1-10 $\mathrm{mM}$ and low aggregation numbers $(2-30)$ near the $\mathrm{CMC}^{1,2}$. They form liquidcrystalline phases only below physiological temperature and at concentrations at least one order of magnitude higher than those in the intestine ${ }^{3}$.

Recent simulations using classical all-atom models of bile salts and explicit water were successfully used to investigate pure bile micelles at bile salt concentrations ranging from 10 to 100 times the $\mathrm{CMC}^{4-6}$. These reports provided important information regarding the shape, size and molecular packing in bile micelles. However, the large system sizes and limited duration inherent to all-atom simulations make them less suited to investigate the equilibrium phase behavior of bile salts under physiological concentrations, or to study the size distribution of bile micelles.

In this paper we report simulations of micelle formation in solutions of pure di- or trihydroxy bile salts at physiological bile-salt and $\mathrm{NaCl}$ concentration. We use a coarse-grained model of bile salts (with glycine or taurine substitution) that is sufficiently detailed to give molecular-scale insight into bile-salt aggregation yet contains sufficiently few degrees of freedom to remain computationally tractable. Our approach allows us to sample the equilibrium behavior of bile-salt solutions over a wide range of concentrations, from below the CMC to about 20 times above it. We analyze the structure and stability of micelles of pure bile salts and identify 
the origin of the unusual surfactant behavior of these substances.

\section{Simulation methodology}

It would be prohibitively expensive to study the phase behavior of bile salts with fully atomistic simulations. We therefore use a coarse-grained model that includes only sufficient detail to distinguish between dihydroxy (called 2OH) and trihydroxy $(3 \mathrm{OH})$ bile salts, as these two salts behave in a qualitatively different fashion. In contrast, all bile salts within these two categories behave qualitatively similarly ${ }^{1}$. In our study we consider only taurine- or glycine-substituted bile salts because these are prevalent in the upper intestinal tract ${ }^{7}$. However, our models do not distinguish between the taurine and glycine substituted compounds.

Experimental results indicate that micelle formation in bile salts differs considerably from that of head-tail surfactants. It seems plausible that the origin of the unusual behavior of bile salts is related to the fact that their molecular architecture is rather different from that of common detergents: the hydrophilic and hydrophobic parts of a bile-salt molecule do not correspond to a "head" or a "tail" of a linear molecule, but to the opposite faces of the concave steroid nucleus. To investigate the effect of molecular architecture we also simulated a hypothetical bile-salt molecule (referred to as $0 \mathrm{OH}$ ) that is identical to the other bile-salt models, except for the fact that there are no hydroxyl groups attached to the steroid nucleus.

Two enhanced sampling techniques are used in this study: Hamiltonian parallel tempering (HPT) and grand-canonical parallel tempering (GCPT). As explained below, HPT is a particularly efficient method to explore the optimal parameterization of a model. We use this method to optimize the key parameter in our model, $\alpha$, the depth of the attraction basin between hydrophobic beads. These HPT simulations are carried out for $2 \mathrm{OH}$ bile salts. The results for $\alpha$ are then transferred to the $3 \mathrm{OH}$ molecule without further optimization. GCPT is well suited to investigate aggregation because it ensures that the osmotic pressure of free monomers is constant throughout the simulation. We use GCPT in our studies of $2 \mathrm{OH}$ and $3 \mathrm{OH}$ bile salts. To investigate aggregation of $0 \mathrm{OH}$ molecules we are forced to revert back to HPT simulations because we found GCPT provides prohibitively inefficient sampling. As detailed descriptions of the simulation techniques that we use can be found in the literature ${ }^{8-14}$ we limit our discussion to a brief summary of the most salient points.

To construct coarse-grained models of the dihydroxy and trihydroxy bile salts, we mostly use a "three-to-one" mapping scheme, where three carbon or nitrogen atoms are grouped into one coarse-grained bead. The exceptions to this rule are 
the hydroxyl groups and the sulfate or carboxyl group at the end of the tail. These are mapped to a single bead. Our coarse-grained model for trihydroxy bile salts is shown in figure 1(a). To aid the reader we also show how this model maps to taurocholate. We emphasize that the model in figure 1(a) is equally representative of other trihydroxy bile salts, such as glycocholate. The region defined by beads 0 to 5 is much less flexible than the tail (beads 5 to 8 ) and corresponds to the steroid nucleus. Connected to this nucleus are three (two, in the case of $2 \mathrm{OH}$ bile salts) hydrophilic beads, corresponding to hydroxyl groups. The flexible tail is composed of beads 5 to 8 with bead 8 being negatively charged. Dihydroxy bile salts (image not shown) are formed by removing bead 11 . The artificial $0 \mathrm{OH}$ molecule is shown in figure 2 .

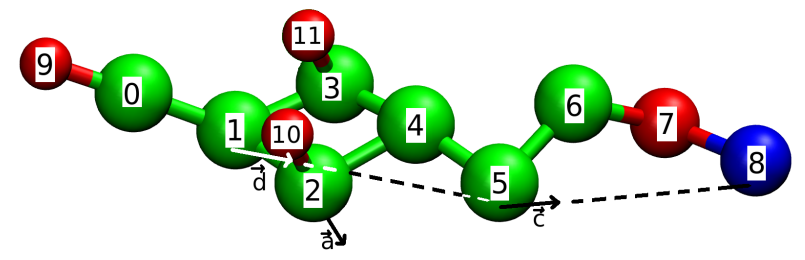

(a)

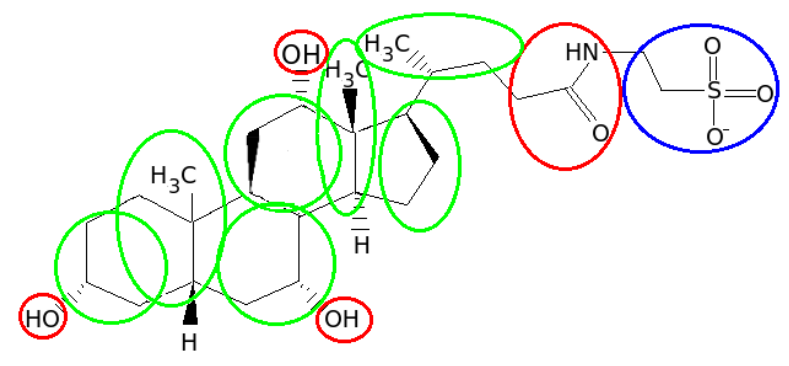

(b)

Figure 1: Correspondence between (a) the coarse-grained model for trihydroxy bile salts and (b) taurocholate. Beads 0 to 6 are hydrophobic, beads 7, 9, 10 and 11 are hydrophilic and bead 8 is charged. The model for dihydroxy bile salts is formed by removing bead 11 . Vectors $\vec{a}, \vec{c}$ and $\vec{d}$ are used to quantify the orientation of molecules as described in section 3.3.

Before describing the potentials that characterize the interactions between beads in our model, we briefly comment on the units used in our simulations. In what follows we use reduced units for computational convenience ${ }^{15}$. To do so we choose appropriate units of length, mass and energy, and express all quantities as multiples of these units. Our unit of length is $\sigma$, of mass is $m$ and of energy is $\epsilon$. It follows that our unit of temperature is $\epsilon / k_{B}$, with $k_{B}$ the Boltzmann 


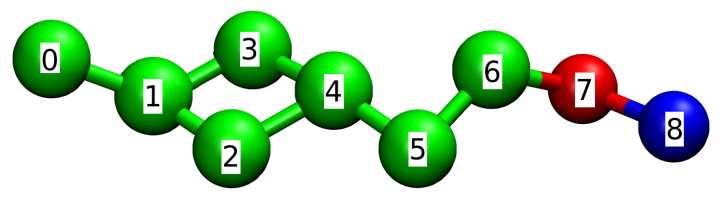

Figure 2: Artificial model $0 \mathrm{OH}$, identical to bile salts but without hydroxyl groups attached to the steroid nucleus.

constant. $\sigma$ is the diameter of the larger beads (beads 0 to 8 ) in figure 1 (a) and corresponds to $\approx 4.0 \AA$, the diameter of the phosphate group, the largest in the molecule. The value of $m \approx 8 \times 10^{-26} \mathrm{~kg}$, is obtained by dividing the mass of a substituted bile salt by the total number of beads in our bile salt model. To define the energy scale we equate the thermal energy scale of our simulations, $\epsilon \times 0.5$, to the real thermal energy scale of a system at $\mathrm{T}=310 \mathrm{~K}, k_{B} \times 310$. Here 0.5 is the reduced temperature at which all simulations are performed. We obtain $\epsilon=8.6 \times 10^{-21} \mathrm{~J} / \mathrm{K}=1.2 \mathrm{kcal} / \mathrm{mol}$, or $\epsilon=2 k_{B} T$.

In our simulations, we do not explicitly model the water nor the added salt ions. Instead, the effect of salt on the behavior of bile salts is modeled through effective interactions between the beads defining the bile molecules. Hydrophobic groups experience an effective attraction when in water, which is the main driving force behind aggregation. In our model this effective attraction is represented by a cosine square potential, $\mathcal{U}_{\text {attr }}(r)$ :

$$
\mathcal{U}_{a t t r}(r)=\left\{\begin{aligned}
4 \alpha \epsilon\left[\left(\frac{\sigma}{r}\right)^{12}-\left(\frac{\sigma}{r}\right)^{6}\right], & r<\sqrt[6]{2} \sigma \\
-\alpha \epsilon \cos ^{2}\left[\frac{\pi(r-\sqrt[6]{2} \sigma)}{2 w_{c}}\right], & \sqrt[6]{2} \sigma \leq r \leq \sqrt[6]{2} \sigma+w_{c} \\
0, & r>\sqrt[6]{2} \sigma+w_{c}
\end{aligned}\right.
$$

We opt for this form of non-bonded potential because it has been used successfully to investigate aggregation of conventional head-tail surfactants ${ }^{16}$. The attraction range $w_{c}$ is set to $1.5 \sigma$. This value was chosen arbitrarily because existing reports on aggregation of standard surfactants using this potential indicate that values of $w_{c}$ between 1 and $1.8 \sigma$ lead to qualitatively similar behavior ${ }^{16} . \sigma$ is the smallest distance at which the potential is zero and $r$ is the actual distance between hydrophobic beads. $\alpha$ determines the depth of the attraction basin and is equal to 0.244 in our simulations. This value of $\alpha$ was determined through Hamiltonian parallel tempering simulations following the procedure described in section 2.1. 
To a first approximation, hydrophilic groups interact equally favorably with water and with each other. We reproduce this behavior by assigning no net attraction or repulsion between them beyond volume exclusion. The interactions between hydrophilic and hydrophobic groups are favorable but much weaker than the effective attraction between hydrophobic groups. For this reason we also assign no net attraction or repulsion between hydrophobic and hydrophilic groups beyond volume exclusion. Volume exclusion for all particles is imposed with a Weeks-Chandler-Andersen potential, $\mathcal{U}_{r e p}(r)$ :

$$
\mathcal{U}_{r e p}(r)=\left\{\begin{aligned}
4 \epsilon\left[\left(\frac{r_{0}}{r}\right)^{12}-\left(\frac{r_{0}}{r}\right)^{6}+\frac{1}{4}\right], & r<\sqrt[6]{2} r_{0} \\
0, & r \geq \sqrt[6]{2} r_{0}
\end{aligned}\right.
$$

As before, $r$ represents the actual distance between two beads. $r_{0}$ is half of the sum of the effective diameter of any two beads and $\sqrt[6]{2} r_{0}$ the distance below which they repel each other. Effective diameters are set at $\sigma$ for all beads except for those with type 9, 10 and, if present, 11 (see figure 1(a)). These beads represent hydroxyl groups, which are smaller than the other groups, and have a diameter of $0.75 \sigma$. Note that $U_{r e p}$ applies also to hydrophobic particles, so the hydrophobichydrophobic interaction is given by $U_{\text {rep }}+U_{\text {attr }}$.

The electrostatic interactions are modeled using a Debye-Hückel potential, $\mathcal{U}_{\text {elect }}$ :

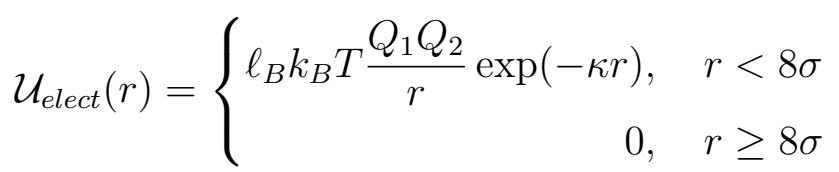

The Debye length, $\kappa^{-1}$, accounts for the effect of the dielectric constant of water and of the presence of $\mathrm{NaCl}$ on the interactions between charged beads. Under physiological conditions, $\kappa^{-1}$ is approximately half the length of the bile salt molecules. We thus assign $\kappa^{-1}=5 \sigma$. The energy scale of the electrostatic interactions is set by the Bjerrum length, $\ell_{B}$, which is the distance at which the electrostatic energy between two particles is equal to the thermal energy scale. In our model, $\ell_{B}=4.5 \sigma$. We define a cutoff radius of $8 \sigma$ because at this distance the interaction energy is much smaller than $k_{B} T . Q_{1}$ and $Q_{2}$ represent the charge of any two particles involved. The only charged particles in our models are those of type 8 (see figure 1(a)), which have a charge of $-1 e$ where $e$ is the absolute charge of the electron.

Bond lengths are enforced through finite extensible nonlinear elastic (FENE) bond potentials of the form

$$
\mathcal{U}_{\text {bond }}(r)=-\frac{1}{2} k_{\text {bond }} \Delta r_{\text {max }}^{2} \ln \left[1-\left(\frac{r}{\Delta r_{\text {max }}}\right)^{2}\right]
$$


$\Delta r_{\max }$ defines the maximum possible extension of the bond and is set at $1.3 \sigma$ for bonds involving hydroxyl groups and $1.5 \sigma$ for all other bonds. The bond stiffness $k_{\text {bond }}$ is set at $40 \epsilon / \sigma^{2}$ for all bonds involving hydroxyl groups, and $30 \epsilon / \sigma^{2}$ for all others. These parameters (coupled with the excluded volume interactions between the beads) ensure that the average bond length is $0.75 \sigma$ for bonds involving hydroxyl groups and $\sigma$ for the remaining ones, with much smaller fluctuations than with other standard bond potentials. Thus, the combination of FENE bonds with excluded volume interactions prevents unphysical crossing of molecules.

The shape and flexibility of the molecules is controlled through angle and dihedral potentials of the form

$$
\mathcal{U}_{\text {angle }}(\theta)=k_{\text {angle }}\left[1-\cos \left(\theta-\theta_{0}\right)\right]
$$

and

$$
\mathcal{U}_{\text {dihed }}(\phi)=k_{\text {dihed }}\left[1+\cos \left(\phi-\phi_{0}\right)\right]
$$

The equilibrium values $\theta_{0}$ and $\phi_{0}$ used are listed in tables 1 and $2 . k_{\text {angle }}$ is set at $0.5 \epsilon$ for the angle potentials involving the particle triads $5 \hat{6} 7$ and $6 \hat{7} 8$, and at $60 \epsilon$ for all other angles. $k_{\text {dihed }}$ is set at $40 \epsilon$ for all dihedral potentials.

Table 1: Equilibrium values, $\theta_{0}$, used in the angle potentials. $\theta_{0}$ is the angle formed by particles $p_{1}, p_{2}$ and $p_{3}, p_{1} \hat{p}_{2} p_{3}$. The particle indices listed correspond to those in figure $1(\mathrm{a})$.

\begin{tabular}{cccc}
\hline$p_{1}$ & $p_{2}$ & $p_{3}$ & $\theta_{0}$ \\
\hline 11 & 3 & 1 & $\pi / 2$ \\
4 & 5 & 6 & $\pi / 2$ \\
10 & 2 & 4 & $\pi / 2$ \\
1 & 3 & 4 & $2 / 3 \pi$ \\
5 & 4 & 3 & $\pi$ \\
9 & 0 & 1 & $\pi$ \\
2 & 1 & 0 & $3 / 4 \pi$ \\
6 & 7 & 8 & $\pi$ \\
5 & 6 & 7 & $\pi$ \\
\hline
\end{tabular}


Table 2: Equilibrium values, $\phi_{0}$, used in the dihedral potentials. $\phi_{0}$ is the angle between the planes defined by the particle triplets $p_{1}, p_{2}, p_{3}$ and $p_{2}, p_{3}, p_{4}$. The particle indices listed correspond to those in figure 1(a).

\begin{tabular}{ccccc}
\hline$p_{1}$ & $p_{2}$ & $p_{3}$ & $p_{4}$ & $\phi_{0}$ \\
\hline 1 & 3 & 4 & 2 & 0 \\
2 & 1 & 3 & 11 & $\pi / 2$ \\
3 & 4 & 2 & 10 & $\pi / 2$ \\
4 & 2 & 1 & 0 & $3 / 4 \pi$ \\
6 & 5 & 4 & 2 & $\pi$ \\
\hline
\end{tabular}




\subsection{Implementation}

All simulations were implemented in the ESPResSo molecular-dynamics simulation package ${ }^{17}$; saved configurations were visualized using $\mathrm{VMD}^{18}$. A constant reduced temperature of $0.5 \epsilon / k_{B}$ is imposed in all simulations through a Langevin thermostat. As mentioned above, this temperature corresponds to the physiological temperature. To integrate the equations of motion, we use the velocity Verlet algorithm with a time step of $0.01 \tau$, where $\tau$ is the reduced unit of time. We make no attempt to relate this unit to real time as we do not report any time-dependent behavior.

Simulations use cubic simulation boxes and periodic boundary conditions. As we typically consider systems at low densities, starting configurations are generated by randomly distributing molecules with identical configuration and orientation in the simulation box. After a small number of integration steps performed with force-capping to resolve possible molecular overlaps, the system is equilibrated until the average number of micelles of each size remains constant. Configurations are saved at every attempt to swap replicas (described in the following subsections) during the production phase. The production phase continues until at least 2000 configurations are obtained.

\subsubsection{Hamiltonian parallel tempering}

Hamiltonian parallel tempering has been described in some detail in several references $^{8-10}$ so here we give only a brief summary of the technique together with the details relevant for our implementation. HPT is analogous to the more widely used temperature parallel tempering ${ }^{11-14}$ but, instead of simulating several replicas of the same system at different temperatures, replicas differ in their Hamiltonian only. The Hamiltonian, $H$, of a system is the sum of the kinetic energy $K(\boldsymbol{p})$ and the potential energy $\mathcal{U}(\boldsymbol{x})$ :

$$
H(\boldsymbol{x}, \boldsymbol{p})=\mathcal{U}(\boldsymbol{x})+K(\boldsymbol{p})
$$

The coordinates and momenta of all particles in the system are represented as $\boldsymbol{x}$ and $\boldsymbol{p}$.

In our simulations, only the value of the parameter $\alpha$ differs between replicas. Note that $\alpha$ modulates principally the depth of the attraction basin between hydrophobic beads without significantly influencing repulsion because the $U_{\text {rep }}$ also applies to hydrophobic particles.

The range of $\alpha$ values used was selected so that the molecules remain as monomers at the lowest $\alpha$ but become stronger micelle formers as $\alpha$ increases. 
Because replicas with adjacent values of $\alpha$ attempt to exchange Hamiltonians periodically during the simulation, the micelle size distribution of replicas with high $\alpha$ can be rapidly sampled. Achieving equally good sampling using simple molecular dynamics or GCPT is prohibitively slow under conditions where molecules are strong micelle formers because the residence time of molecules in micelles is long. Hamiltonian parallel tempering thus has two advantages: in a single simulation one can simultaneously test a wide range of values of a parameter and ensure that canonical equilibrium distributions are correctly sampled at each replica. In this work we use HPT simulations during the parameterization stage, to determine the optimal value of $\alpha$, and again during the production stage, to investigate aggregation of $0 \mathrm{OH}$ molecules.

During the parameterization stage we perform simulations of $2 \mathrm{OH}$ bile salts only. We use a total of 26 replicas with $\alpha_{\min } \leq \alpha \leq \alpha_{\max }=0.19 \leq \alpha \leq 0.28$ distributed according to

$$
\alpha_{i}=\alpha_{\min }+\frac{\alpha_{\max }-\alpha_{\min }}{\text { no. of replicas }-1} i \quad i=0,1 \cdots 25
$$

All replicas have identical number density $\left(4.5 \times 10^{-4}\right.$ molecules $\left./ \sigma^{3}\right)$ and number of molecules (50). HPT is also used to investigate aggregation of $0 \mathrm{OH}$ molecules, for the reasons described above. In these simulations we used 32 replicas each with 70 molecules and $0.19 \leq \alpha \leq 0.248$.

The exchange of Hamiltonians between consecutive replicas is attempted every 20000 steps for $2 \mathrm{OH}$ or 100000 steps for $0 \mathrm{OH}$ simulations. More frequent attempts proved inefficient because they resulted in the same configurations swapping back and forward between replicas, not in enhanced sampling of configurations. The probability of accepting any given attempt so that the extended system of replicas goes from state $\left\{H_{i}\left(\boldsymbol{x}_{e}, \boldsymbol{p}_{e}\right), H_{i+1}\left(\boldsymbol{x}_{f}, \boldsymbol{p}_{f}\right)\right\}$ to state $\left\{H_{i+1}\left(\boldsymbol{x}_{e}, \boldsymbol{p}_{e}\right), H_{i}\left(\boldsymbol{x}_{f}, \boldsymbol{p}_{f}\right)\right\}$ is

$$
\min [1, \exp (-\Delta)]
$$

with

$$
\Delta=\beta\left[\mathcal{U}_{i+1}\left(\boldsymbol{x}_{e}\right)-\mathcal{U}_{i}\left(\boldsymbol{x}_{e}\right)+\mathcal{U}_{i}\left(\boldsymbol{x}_{f}\right)-\mathcal{U}_{i+1}\left(\boldsymbol{x}_{f}\right)\right]
$$

Here $\beta=1 / T \epsilon^{-1} . H_{i}\left(\boldsymbol{x}_{e}, \boldsymbol{p}_{e}\right)$ represents a replica with coordinates and momenta $\boldsymbol{x}_{e}, \boldsymbol{p}_{e}$ for which the parameter $\alpha$ takes the value $\alpha_{i}$. The subscripts $i, i+1, e$ and $f$ were added for clarity, to emphasize that the two replicas have distinct values of $\alpha$, particle coordinates and momenta. Note that the particle momenta do not influence the acceptance probability and that $\mathcal{U}$ need only include the part of the Hamiltonian that changes between replicas $\left(\mathcal{U}_{\text {attr }}\right.$ in our simulations).

From simulations of $2 \mathrm{OH}$ bile salts we identify the value of $\alpha$ for which the system forms micelles and, in particular, the value that yields the critical micelle 
concentrations and micellar sizes that are in best agreement with the available experimental information on dihydroxy bile salts: $\alpha=0.244$. We show in section 3 that the same value is found to lead to the correct aggregation behavior of trihydroxy bile salts without the need of re-parametrization.

\subsubsection{Grand canonical parallel tempering}

GCPT is the method of choice to investigate aggregation. In GCPT each replica is simulated at the same temperature and volume but (unlike for HPT) at a different chemical potential ${ }^{15}$. Thus, a single simulation can be used to investigate aggregation over a range of concentrations. The chemical potential at each replica is enforced through frequent molecule insertions and deletions. The GCPT scheme is an efficient way to sample equilibrium distributions in our system because the number, size and stability of micelles decrease with decreasing chemical potential. The frequent insertion and deletion of molecules is sufficient for replicas at low chemical potential to rapidly reach equilibrium. Because replicas with adjacent chemical potentials periodically attempt to exchange them during the simulation, replicas at higher chemical potentials can also efficiently sample their equilibrium distributions.

Between 20 and 100 insertions and deletions are attempted between tries to swap chemical potentials in simulations of $2 \mathrm{OH}$ and $3 \mathrm{OH}$. The probability of accepting a molecule insertion or deletion is given by ${ }^{15}$

$$
P(N \rightarrow N+1)=\min \left[1, \frac{V \rho_{i d}}{(N+1)} \exp \left(-\beta\left[\mathcal{U}_{\text {inter }}\left(\boldsymbol{x}^{N+1}\right)-\mathcal{U}_{\text {inter }}\left(\boldsymbol{x}^{N}\right)\right]\right)\right]
$$

and

$$
P(N \rightarrow N-1)=\min \left[1, \frac{N}{V \rho_{i d}} \exp \left(-\beta\left[\mathcal{U}_{\text {inter }}\left(\boldsymbol{x}^{N-1}\right)-\mathcal{U}_{\text {inter }}\left(\boldsymbol{x}^{N}\right)\right]\right)\right]
$$

$N$ is the number of molecules in the system before the attempted insertion or deletion, $V$ is the volume of the simulation box, $\mathcal{U}_{\text {inter }}\left(\boldsymbol{x}^{N}\right)$ is the intermolecular potential energy of a system with $N$ molecules. The superscripts $N, N+1, N-1$ are not exponents; they were added for clarity, to emphasize the change in number of molecules. $\rho_{i d}$ is the molecular number density of the ideal gas reservoir used to set the chemical potential of our system. $\rho_{i d}$ is thus entirely analogous to the fugacity and we refer to it as such. The ensemble of configurations of the inserted molecules are generated in a separate simulation of a single bile salt molecule.

The fugacity of the replicas in our simulations ranges from below the CMC to up to 20 times above it. For $2 \mathrm{OH}$ and for $3 \mathrm{OH}$ bile salts we used $\rho_{i d, \min }=2.2 \times 10^{-4}$ 
and $\rho_{i d, \max }=0.01$ molecules $/ \sigma^{3}$ and 52 replicas. The values of the fugacity are distributed following an arithmetic progression analogous to that in equation 8.

Attempts to swap fugacities between two replicas are made every 20000 steps for all simulations. The probability of accepting any given swap so the ex-

tended system of replicas goes from state $\left\{\left[\left(\boldsymbol{x}_{e}, \boldsymbol{p}_{e}\right), \rho_{i d, i}\right],\left[\left(\boldsymbol{x}_{f}, \boldsymbol{b}_{f}\right), \rho_{i d, i+1}\right]\right\}$ to state $\left\{\left[\left(\boldsymbol{x}_{e}, \boldsymbol{p}_{e}\right), \rho_{i d, i+1}\right],\left[\left(\boldsymbol{x}_{f}, \boldsymbol{b}_{f}\right), \rho_{i d, i}\right]\right\}$ is

$$
\min \left[1,\left(\rho_{i d, i}\right)^{N_{f}-N_{e}}\left(\rho_{i d, i+1}\right)^{N_{e}-N_{f}}\right]
$$

where $N_{e}$ and $N_{f}$ are the number of molecules in the two replicas. As above, the indices $i, i+1, e$ and $f$ were added for clarity, to emphasize that the two replicas have distinct fugacities, coordinates and momenta.

\section{Results and discussion}

\subsection{Critical micellar concentrations, aggregation numbers and micellar shape}

Having determined the optimal value of $\alpha$ from canonical HPT simulations at concentrations near the experimental values of the CMC, we proceed to investigate aggregation of $2 \mathrm{OH}$ and $3 \mathrm{OH}$ bile salts at various concentrations using GCPT. To identify micelles we define a connectivity criterion between neighboring molecules: molecules are considered a part of the same aggregate if their centers of mass are within $3 \sigma$ of each other. This criterion is chosen based on the radial distribution function $(\mathrm{G}(\mathrm{r}))$ of the centers of mass $(\mathrm{COM})$ of $2 \mathrm{OH}$ molecules, shown in figure 3 for a bile salt concentration near the CMC. The form of the curve for distances $<3 \sigma$ does not depend on bile salt concentration. Small variations in the threshold distance used to define nearest neighbors do not affect our results. 


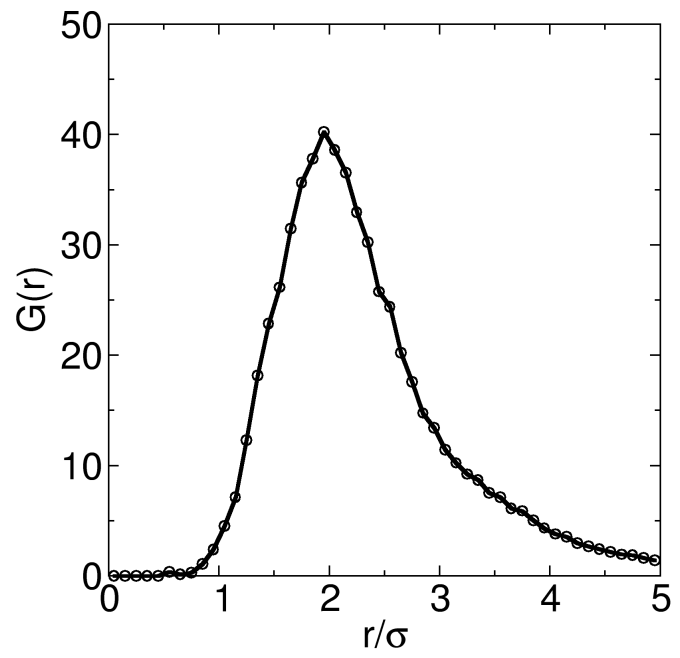

Figure 3: Radial distribution function, G(r), of the centers of mass of molecules of $2 \mathrm{OH}$ bile salts at the CMC. 
The CMC is an important property of a surfactant, so it is useful to evaluate how well our models reproduce it. The experimentally determined CMC is the lowest concentration at which the behavior of the surfactant solution changes, indicating that the system response begins to be dominated by micelles and not monomers. The definition of the $\mathrm{CMC}$ is not unique: different experimental methods normally lead to (slightly) different values ${ }^{19}$. Here we define the CMC as the concentration at which $50 \%$ of the molecules exist in aggregates.

In table 3 we present the value of the CMC obtained for $2 \mathrm{OH}$ bile salts. Analogous information is given in table 4 for $3 \mathrm{OH}$ bile salts. Volume fraction is the natural way to express concentration in simulations that use reduced units because concentrations are then independent of the mapping of $\sigma$ to S.I. units. Because experimental CMCs are normally reported in units of mole per unit volume, we report them both in volume fraction and mM. The experimentally determined CMCs for various conjugated dihydroxy bile salts are also presented in both units to faciliate comparisons.

Our minimal model slightly overestimates the CMC. This small discrepancy is not surprising as during model parameterization we opted to enhance agreement of aggregation numbers with experiment at the expense of agreement of CMCs. Nevertheless, the present model distinguishes between di- and trihydroxy bile salts: in agreement with experiment, our predicted CMC for trihydroxy bile salts is higher than for dihydroxy ones. The fact that the model appears to overestimate the CMC implies only that care should be taken when using it to predict CMCs of mixes of bile salts and other molecules. This limitation does not impact the model's applicability in studies of micelle structure and formation mechanism. 
Table 3: Comparison of CMC values obtained in the present work for dihydroxy bile salts to those from experimental studies of dihydroxy salts taurodeoxycholate (TDC) and taurochenodeoxycholate (TCDC).

\begin{tabular}{lllll}
\hline Bile salt & CMC, $\mathrm{v} / \mathrm{v}^{* *}$ & $\mathrm{CMC}, \mathrm{mM}^{*}$ & Method & Ref. \\
\hline 2OH & 0.0024 & 11 & this work & \\
TCDC & 0.0021 & 7.0 & dye solubilization & 20 \\
TCDC & 0.0012 & 4.0 & dye solubilization & 20 \\
TCDC & 0.0006 & 2.0 & spin label & 21 \\
TCDC & 0.0005 & 1.8 & dye titration & 22 \\
TDC & 0.0018 & 6.0 & dye solubilization & 20 \\
NaTDC & 0.0010 & 3.2 & spin label & 21 \\
\hline
\end{tabular}

* The CMC from this work was calculated assuming $\sigma=4.0 \AA$.

$* *$ Experimental values were converted from $\mathrm{mM}$ assuming

that the molecular volume of bile salts is $500 \AA^{3} /$ molecule $^{1,2}$.

Table 4: Comparison of CMC values obtained in the present work for trihydroxy bile salts to those from experimental studies of trihydroxy salt taurocholate.

\begin{tabular}{llll}
\hline CMC, v/v** & ${\text { CMC }, \mathrm{mM}^{*}}^{*}$ Method & Ref. \\
\hline 0.0043 & 17 & this work & 20 \\
0.0027 & 9.0 & dye solubilization & 20 \\
0.0018 & 6.0 & surface tension & 21 \\
0.0015 & 5.0 & spin label & 20 \\
0.0012 & 4.0 & dye solubilization & 1 \\
0.0008 & 2.7 & spectral shift of rhodamine $6 \mathrm{G}$ & 1 \\
\hline * The CMC from this work was calculated assuming $\sigma=4.0 \AA$ \\
** Experimental values were converted from $\mathrm{mM}$ assuming \\
that the molecular volume of bile salts is $500 \AA^{3} /$ molecule ${ }^{1,2}$.
\end{tabular}


To allow further direct comparison with experiment we compute another important property of surfactants, the average aggregation number. The average aggregation number may include or exclude monomers; here we opt to calculate the aggregation number excluding monomers, $\left\langle n_{\text {excl. }}\right\rangle$ :

$$
\left\langle n_{\text {excl. }}\right\rangle=\frac{\sum_{n=2}^{N} n C_{n}}{\sum_{n=2}^{N} C_{n}}
$$

Here $C_{n}$ represents the concentration of micelles with aggregation number $n$ averaged over all saved configurations of a replica at a given fugacity from GCPT simulations.

Figure $4(\mathrm{a})$ shows $\left\langle n_{\text {excl. }}\right\rangle$ as a function of concentration from our simulations. In figure 4(b) we reproduce the average aggregation number excluding monomers obtained from gel filtration chromatography of dihydroxy and trihydroxy bile salts $^{23}$. The bile salt concentration in these graphs is given as a multiple of the CMC to facilitate comparison, because (as we mentioned above) our simulations overestimate the CMC. We note that the experimental values of $\left\langle n_{\text {excl. }}\right\rangle$ have an associated uncertainty of at least 30\%. Comparison of these two figures thus confirms that, if the concentrations are referred to the CMC, the micellar aggregation numbers from our simulations agree fairly well with experiments on similar bile salts. Table 5 shows average aggregation numbers for various conjugated dihydroxy and trihydroxy bile salts. The differences between the various dihydroxy (or trihydroxy) salts are small, confirming that our models give insight into the common behavior of conjugated di- and trihydroxy bile salts and not just taurodeoxycholate and taurocholate. 


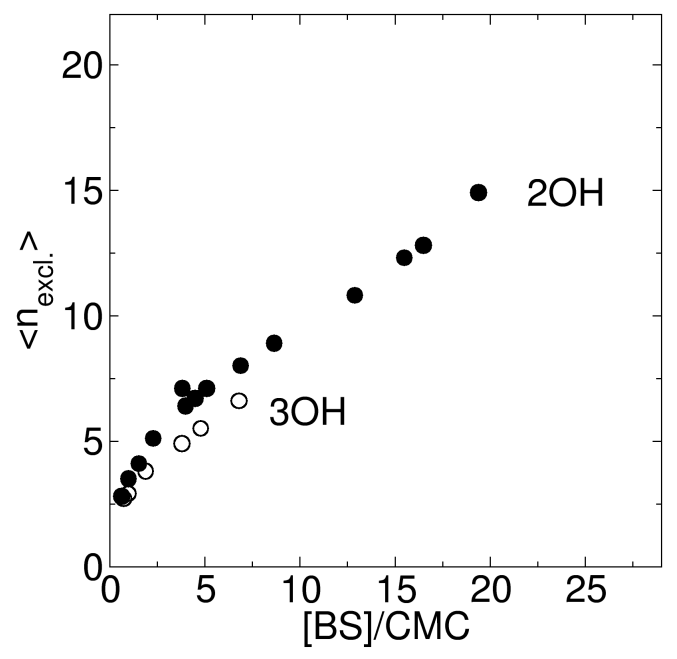

(a)

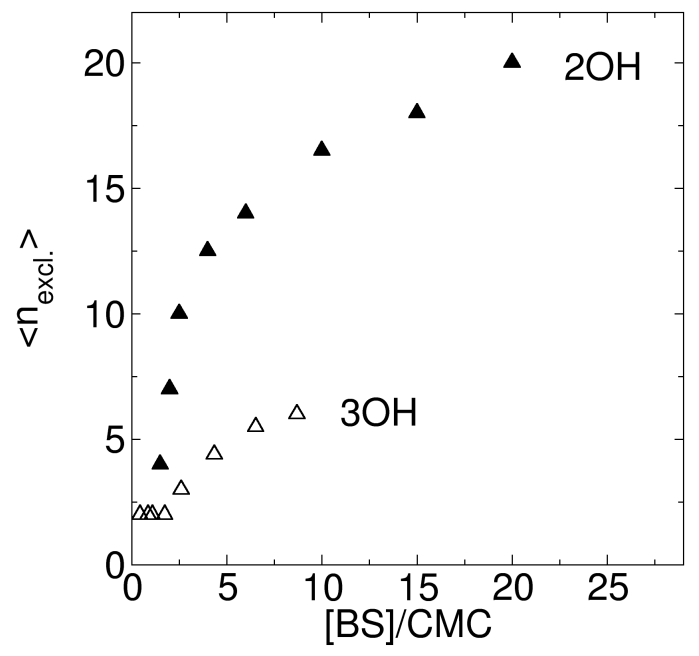

(b)

Figure 4: Aggregation number excluding monomers $\left(\left\langle n_{\text {excl. }}\right\rangle\right)$ as a function of the total bile salt concentration, for dihydroxy and trihydroxy bile salts from (a) our simulations and from (b) chromatography experiments with taurodeoxycholate $(2 \mathrm{OH})$ and taurocholate $(3 \mathrm{OH})$ (adapted from Funasaki et al. ${ }^{23}$ ). 
Table 5: Aggregation numbers of dihydroxy and trihydroxy bile salts from experiments at $36{ }^{\circ} \mathrm{C}$. From ref. ${ }^{24}$ in $^{1}$, pp. 318. GCDC = glycochenodeoxycholate, $\mathrm{TCDC}=$ taurochenodeoxycholate, $\mathrm{TDC}=$ taurodeoxycholate, GDC $=$ glycodeoxycholate, $\mathrm{GC}=$ glycocholate, $\mathrm{TC}=$ taurocholate .

\begin{tabular}{lcc}
\hline Bile salt & Type & Agg. no. \\
\hline GCDC & $2 \mathrm{OH}$ & 18.2 \\
TCDC & $2 \mathrm{OH}$ & 15.2 \\
TDC & $2 \mathrm{OH}$ & 18.0 \\
GDC & $2 \mathrm{OH}$ & 16.2 \\
\hline GC & $3 \mathrm{OH}$ & 5.6 \\
TC & $3 \mathrm{OH}$ & 4.5 \\
\hline
\end{tabular}


We characterize the shape of micelles by computing the ratios of the principal moments of inertia, $I_{1} / I_{2}$ and $I_{2} / I_{3}\left(I_{1}<I_{2}<I_{3}\right)$, of micelles of various sizes. If the micelles are approximately spherical, $I_{1} / I_{2} \approx I_{2} / I_{3} \approx 1$, if they are disklike $I_{1} / I_{2} \approx 1$ and $I_{2} / I_{3}<1$ and if they are rod-like, $I_{1} / I_{2}<1$ and $I_{2} / I_{3} \approx$ 1. We present our results in the form of two-dimensional normalized frequency histograms of $I_{1} / I_{2}$ vs. $I_{2} / I_{3}$ for $2 \mathrm{OH}$ bile salts in figure 5 . The smaller micelles are roughly spherical but larger ones tend to be prolate for both $2 \mathrm{OH}$ and $3 \mathrm{OH}$ (results not shown) bile salts. This is in line with results from light scattering experiments of tauro-substituted bile salts ${ }^{25}$. Fully atomistic simulations of glycine-substituted bile salts ${ }^{6}$ found evidence for slightly oblate micelles $\left(I_{1} / I_{2} \approx 0.9\right.$ and $I_{2} / I_{3} \approx$ $0.8)$. However, these aspect ratios are not significantly different from the results reported in figure 5 . 


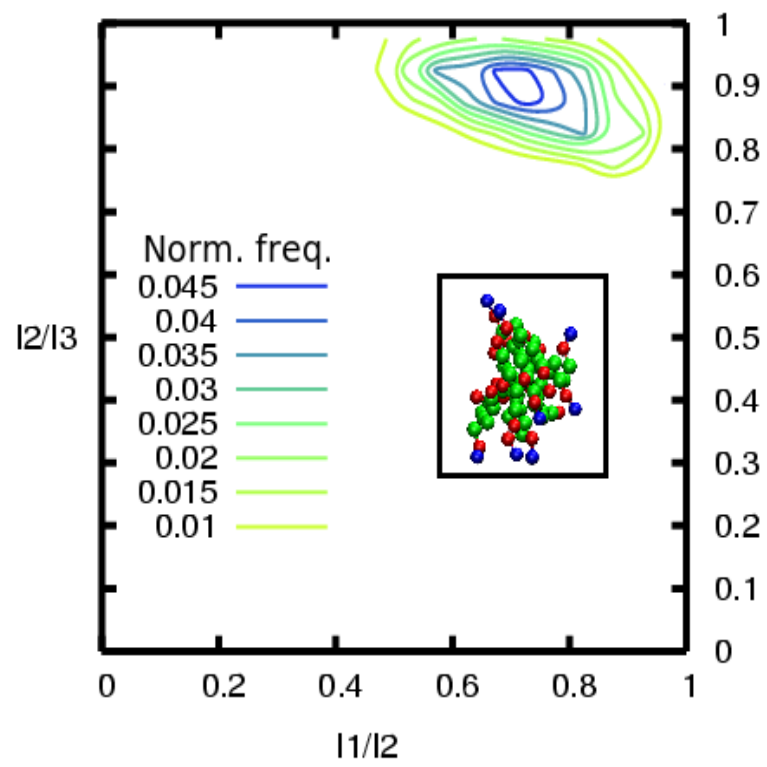

(a)

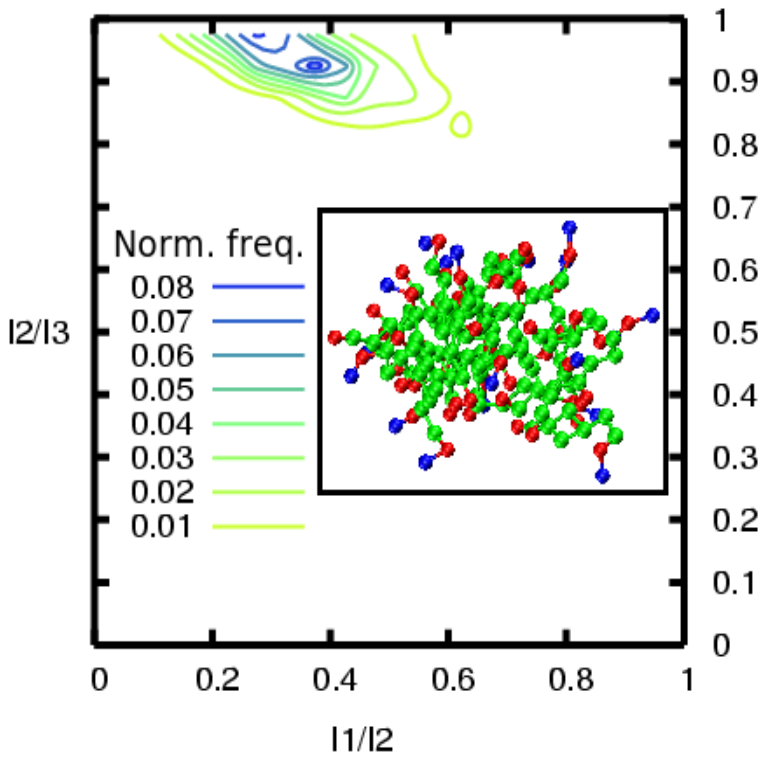

(b)

Figure 5: Normalized frequency histogram of the ratios $I_{1} / I_{2}$ and $I_{2} / I_{3}$ of the principal moments of inertia of micelles of $2 \mathrm{OH}$ bile salts (a) with 10 molecules and (b) with 25 molecules. The insets show an example of small and large micelles. 
Small-angle neutron scattering experiments may be used to infer micellar shape. However, a direct interpretation of the experimental data is not straightforward. Rather, the common procedure is to fit the predictions of simple models to the experimental data. Here we present theoretical neutron scattering curves for dihydroxy bile salts which can be compared with experiment and we suggest a model useful for the treatment of experimental data.

The radially averaged coherent differential cross-section per molecule, $I_{C}^{\prime}(q)$, at a given bile salt concentration $C$, is given by

$$
I_{C}^{\prime}(q)=\left\langle\frac{1}{N} \sum_{i=1}^{N * v} \sum_{j=1}^{N * v} b_{i} \cdot b_{j} \frac{\sin \left(q \cdot r_{i j}\right)}{q \cdot r_{i j}}\right\rangle
$$

where $N$ is the number of molecules in the system, $v$ is the number of beads per molecule, $b$ is the scattering length of each bead, $q$ is the modulus of the scattering vector and $r_{i j}$ is the distance between beads $i$ and $j$. The scattering length of each bead is calculated as the average over the scattering lengths of all the atoms that compose that bead following the mapping scheme shown in figure 1 . Only $q$ values compatible with the size of the simulation box may be used, so $q=2 \pi \cdot k / L$ with $L$ the width of the simulation box and $k=1,2, \cdots$. The average is over all recorded configurations from simulations at a given concentration. To facilitate comparison between curves for different bile salt concentrations, we normalize $I_{C}^{\prime}(q)$ by the concentration, thus obtaining $I_{C}(q)$ according to

$$
I_{C}(q)=\frac{C}{C^{\prime}} I_{C}^{\prime}(q)
$$

Here $C^{\prime}$ is the smallest bile concentration for which $I(q)$ is calculated.

Our results, given in figure 6 , suggest that local order increases with bile salt concentration. This is indicated by the increasing depth of the well in region $\mathrm{B}$ of figure 6 , which corresponds to a distance of 7-8 $\sigma$.

In section 3.3 we will show that the charged group stays preferentially on the outside of the micelle and that the inside of the micelle contains both hydrophilic and hydrophobic groups. Based on this we recalculate $I_{C}(q)$ by considering that the bile molecule has only two types of beads. Beads 7 and 8 are assigned the average scattering length between them, and all remaining beads are assigned their corresponding average scattering length. This corresponds to a core-shell model where beads 7 and 8 make up the outer shell of micelles and all others belong to the core. Our results (not shown) indicate that this model reproduces well the true $I(q)$ shown in figure 6 . This suggests that a core-shell model may be appropriate for the analysis of experimental results. 


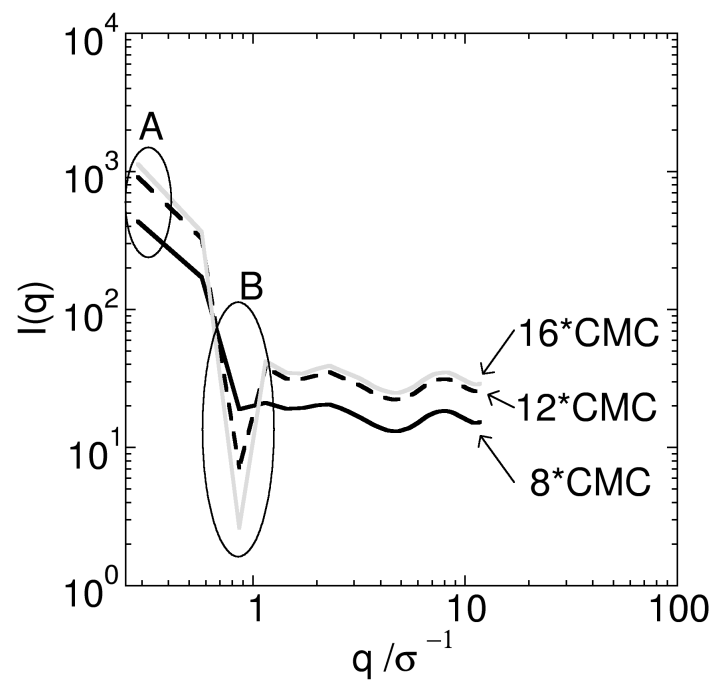

Figure 6: Theoretical neutron scattering coherent differential cross-section, $I(q)$, for dihydroxy bile salts at concentrations 8,12 and 16 times higher than the critical micellar concentration. In region $\mathrm{A}, q \approx 0.05-0.1 \AA^{-1}$ and in region $\mathrm{B}$ $q \approx 0.15-0.3 \AA^{-1}$. 


\subsection{Micelle formation}

Micelle formation is typically a cooperative process: it requires the simultaneous, collective organization of a well defined number of surfactants to overcome the free energy barrier for micelle formation. We investigate the cooperativity of micelle formation by computing the probability of finding micelles of any given size at a given bile salt concentration. This micelle size distribution, $P(n)$, is calculated as

$$
P(n)=\frac{\left\langle N_{n}\right\rangle}{\sum_{i=1}^{\infty}\left\langle N_{i}\right\rangle}
$$

where $N_{n}$ (or $N_{i}$ ) represent micelles of size $n(i)$ and the averages are calculated over all saved configurations for the replica of interest.

Figure 7 shows the micelle size distribution for both dihydroxy and trihydroxy bile salts, at the $\mathrm{CMC}$ and at $\approx 8 \times \mathrm{CMC}$. This distribution indicates that the micelle formation process is poorly cooperative. Also, the high CMC indicates that bile salts are simply not very good micelle formers. At the CMC (figure 7(a)), $P(n)$ does not present the typical minimum followed by a maximum near the average aggregation number that is often exhibited by conventional head-tail surfactants. Rather, it decays monotonically from the maximum at $n=1$. The somewhat large variation in reported CMCs of bile salts in the literature is compatible with this observation. The typical $P(n)$ shape associated with micelle formation of simple surfactants (see e.g. Pool et al. ${ }^{26,27}$ ) only appears at higher concentrations (see figure $7(\mathrm{~b})$ ). Three aspects of bile salt behavior can be inferred from this figure. Firstly, bile salts form highly polydisperse micelles. Polydispersity is defined as the standard deviation of the $P(n)$ distributions excluding monomers, normalized by their mean ${ }^{25}$. Polydispersity is $\approx 50 \%$ for both $2 \mathrm{OH}$ and $3 \mathrm{OH}$ bile salts, which is higher than what is observed in conventional head-tail surfactants like SDS. Secondly, dimers are abundant at concentrations much higher than the CMC, indicating that they are unusually stable. And finally, the low cooperativity in micelle formation is apparent also at high concentrations, in the shallow minimum separating the micelles from the monomers. This feature confirms that the free energy barrier to micelle disassembly is still comparable to the thermal energy scale at $\approx 8 \mathrm{CMC}$, thus enabling bile micelles to assemble and disassemble rapidly. This characteristic is even more marked for $3 \mathrm{OH}$ bile salts.

To suggest possible reasons behind the small size, high polydispersity and low cooperativity of formation of bile micelles, we perform simulations using molecules identical to bile salts in all aspects but without any $\mathrm{OH}$ groups (called $0 \mathrm{OH}$ ). This system has no experimental parallel and serves only as an artificial control, allowing us to identify the source of the behavior of $2 \mathrm{OH}$ and $3 \mathrm{OH}$ bile salts. We 
find that the $\mathrm{CMC}$ of $0 \mathrm{OH}$ is considerably lower than either $2 \mathrm{OH}$ or $3 \mathrm{OH}$ bile salts - $0.0007 \mathrm{v} / \mathrm{v}$ versus 0.002 or $0.004 \mathrm{v} / \mathrm{v}$ - indicating that $0 \mathrm{OH}$ molecules are less soluble than $2 \mathrm{OH}$ or $3 \mathrm{OH}$. This is expected since $0 \mathrm{OH}$ molecules contain no hydrophilic $\mathrm{OH}$ groups.

The micelle size distribution for $0 \mathrm{OH}$ is shown in figure 8 for a concentration $\approx 4 \times \mathrm{CMC}$. The main graph does not include the probability of finding monomers, which is much higher than the probability of finding micelles. The full $P(n)$ distribution is shown in the inset in logarithmic scale. We compare this distribution with the corresponding ones obtained for $2 \mathrm{OH}$ and $3 \mathrm{OH}$ molecules, shown in figure 7(b). Figure 8 indicates that $0 \mathrm{OH}$ molecules are much stronger micelle formers than either $2 \mathrm{OH}$ and $3 \mathrm{OH}$ : the free-energy barrier to micelle disassembly is high, as indicated by the deep $P(n)$ minimum separating monomers and aggregates with $n<5$ from the larger aggregates. Micelle formation is more cooperative for $0 \mathrm{OH}$ than for either $2 \mathrm{OH}$ or $3 \mathrm{OH}$, as attested by the larger size of $0 \mathrm{OH}$ micelles.

Our coarse-grained model reproduces differences in micelle size between trihydroxy and dihydroxy bile salts observed in experiments and all-atom simulations ${ }^{1,5}$ : trihydroxy bile salts form smaller micelles than dihydroxy salts at similar concentrations. Furthermore, the $0 \mathrm{OH}$ molecules form larger micelles than either $2 \mathrm{OH}$ or $3 \mathrm{OH}$. As our model does not account for hydrogen bonding, our findings suggest that hydrogen bonds are not necessary to stabilize micelles. Such a mechanism was initially proposed by Small ${ }^{1}$, who postulated that only smaller (called primary) micelles form by hydrophobic interactions, with hydrogen bonds being indispensable for the formation of larger ("secondary") micelles by hydrogen bond interactions between primary micelles ${ }^{1}$. Subsequent experimental work ${ }^{28}$ and simulations using united atom models in explicit water ${ }^{4,5}$ found that hydrogen bonds were abundant in both primary and secondary bile micelles. The presence of hydrogen bonds was interpreted as evidence that they are indispensable for micelle formation. While it seems certain that hydrogen bonds form in bile micelles and are likely to stabilize certain micelle configurations relative to others, our results suggest that hydrogen bonds do not drive aggregation. They are nevertheless crucial for aggregation, making the bile salts more soluble and bile micelles small. In the following section we show that hydrophobic groups are exposed at the surface of both small and large micelles, enabling micellar growth in the absence of intramicellar hydrogen bonds. 


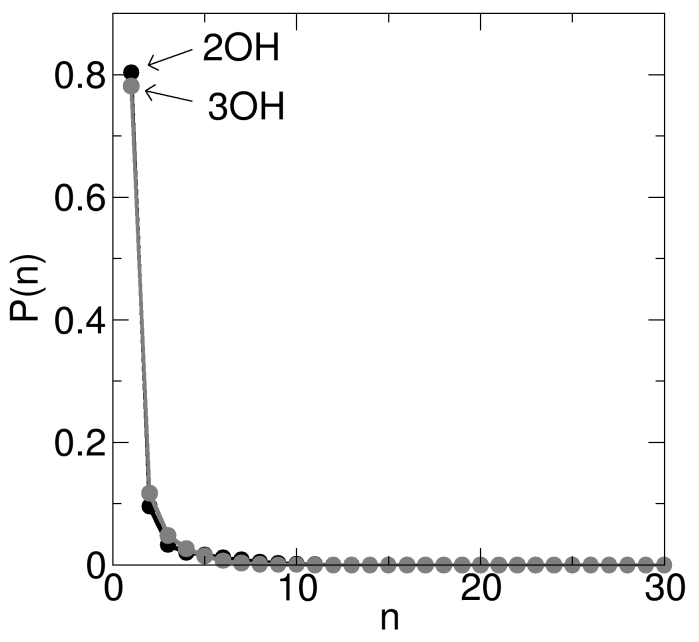

(a)

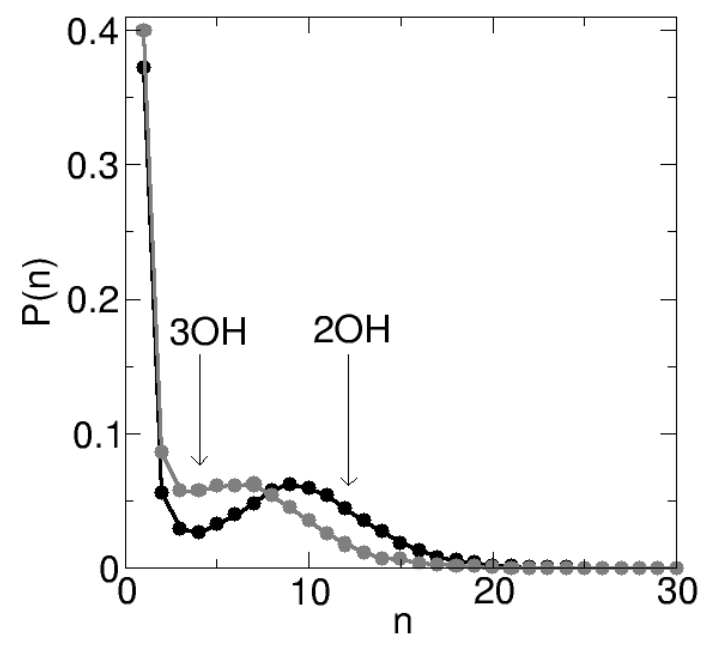

(b)

Figure 7: Micelle size distribution $P(n)$ for dihydroxy (black) and trihydroxy (gray) bile salts (a) at the $\mathrm{CMC}$ and (b) at $8 \times \mathrm{CMC}$. 


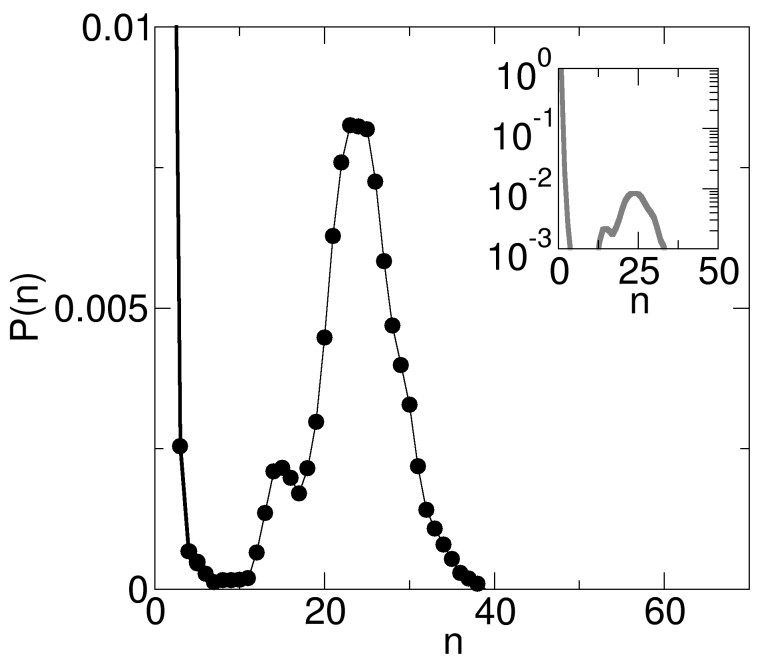

Figure 8: Micelle size distribution $P(n)$ for $0 \mathrm{OH}$ at $\approx 4 \times \mathrm{CMC}$. The simulations of this model system are very time consuming and hence the raw data for $P(n)$ are noisy. The $P(n)$ shown in this figure has been smoothed by averaging over 3 bins at a time. The inset shows the same data as the main graph using a logarithmic scale for the y-axis. 


\subsection{Structural properties of bile micelles}

The coarse-grained inner structure of a micelle is well described by the local density of each type of bead. In figure 9 we show the radial densities for hydrophilic, hydrophobic and charged beads for spherical and prolate micelles of $2 \mathrm{OH}$ bile salts. The local density is given as a function of the distance to the center of mass of the micelle for spherical micelles or, in the case of prolate ones, to the micelle's long axis. The $3 \mathrm{OH}$ bile salts show similar behavior (not shown). As expected charged groups remain preferentially on the outside of the micelles. As mentioned in the previous section, the hydrophobic parts of the molecule are not fully shielded from the water: they are often found at the surface of the micelle. Titration experiments using cholate and deoxycholate and all-atom simulations are in line with our observations ${ }^{4,5,29}$. Interestingly, bile micelles do not have a purely hydrophobic core. Instead we find that the density of hydrophilic groups inside the micelle is approximately constant. 


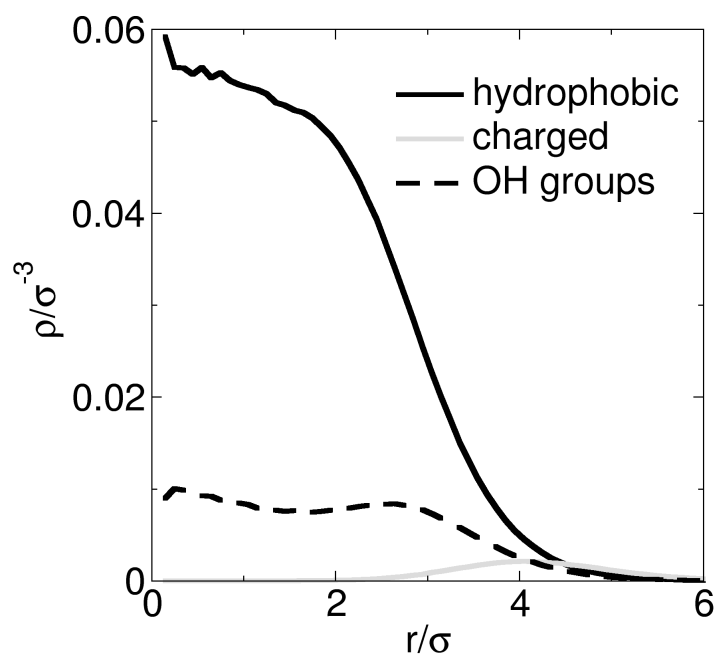

(a)

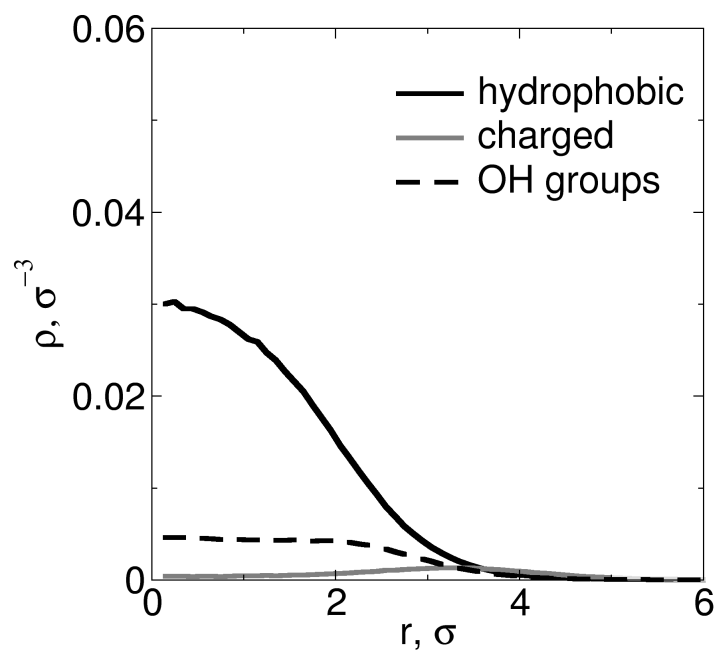

(b)

Figure 9: Radial number density, $\rho$, of hydrophilic, hydrophobic and charged beads for (a) spherical micelles $(I 1 / I 2>0.5$ and $I 2 / I 3>0.7)$ and (b) prolate micelles $(0.3<I 1 / I 2<0.4$ and $I 2 / I 3>0.7)$ of $2 \mathrm{OH}$ bile salts. 
The fact that the density of hydrophilic groups inside the micelle is approximately constant suggests that bile molecules are not strongly oriented. To quantify this we determined the degree to which bile molecules are oriented radially. More precisely, we looked at the orientational ordering of three vectors characterizing the 3D orientation of the bile molecule: the first vector $(\vec{a})$ is a unit vector perpendicular to the steroid nucleus (see figure 1(a)); the second, $(\vec{c})$ is the unit vector associated with the direction of the tail of the molecule (from bead 5 to 8 in the same figure); the third vector $(\vec{d})$ is a unit vector associated with the direction of the long axis of the steroid nucleus (from bead 1 to 5 in the same figure). If the tail of bile molecules was rigid, vectors $\vec{c}$ and $\vec{d}$ would be approximately parallel. Because the tail is not rigid, it is important to quantify the radial orientation of both vectors. We calculate the orientational distribution of $\vec{r} \cdot \vec{a}, \vec{r} \cdot \vec{c}$ and $\vec{r} \cdot \vec{d}$, where $\vec{r}$ is the unit vector in the direction that joins the center of mass of the micelle to that of a given bile-salt molecule (see figure 10).

If bile molecules would be orientationally ordered, $\vec{c} \cdot \vec{r}$ and $\vec{d} \cdot \vec{r}$ would peak at 1 and $\vec{r} \cdot \vec{a}$ at 0 . Instead we observe very broad distributions, indicating that bile salts may take a wide range of orientations in spherical micelles. This indicates that the typical representations of small bile micelles (for examples see Padmanabhan et al. ${ }^{1,2}$ ) represent a small subset of

possible orientations. Our static image of bile micelles must be replaced by a fluid one. 


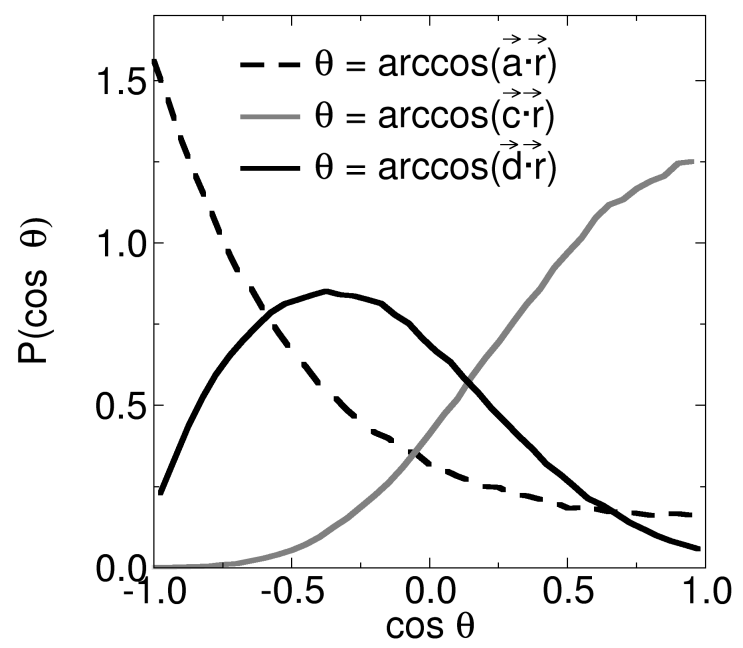

Figure 10: Distribution of the angle formed by the $\vec{a}, \vec{c}$ and $\vec{d}$ vectors with the unit vector $\vec{r}$ associated with the position of each molecule relative to the (spherical) micelle's center of mass. Results for $2 \mathrm{OH}$ bile salts. 
Our results suggest bile micelles have reduced long range order at all concentrations investigated, but local order may still exist. We quantify local order by the degree to which neighboring bile-salt molecules align. Again, we use the unit vector $\vec{d}$ (aligned with the long axis of the molecule) but, in addition, we define a unit vector, $\vec{a}$, perpendicular to the plane of the steroid nucleus and pointing in the direction opposite bead 10 in figure 1(a). Figure 11 shows the distribution of $\overrightarrow{a_{i}} \cdot \overrightarrow{a_{j}}$ and $\overrightarrow{d_{i}} \cdot \overrightarrow{d_{j}}$ vectors for all pairs $\{i j\}$ of first neighbors for dimers $(\mathrm{n}=2)$ and larger micelles of $2 \mathrm{OH}$ bile salts. Results for micelles of different sizes and for $3 \mathrm{OH}$ bile salts are similar (not shown). As the figure shows, the tails of neighboring molecules may be parallel or anti-parallel $\left(\vec{d}_{i} \cdot \vec{d}_{j}\right.$ peaking at 1 or -1$)$. Neighboring molecules preferentially orient their steroid rings so that $\vec{a}_{i} \cdot \vec{a}_{j}$ peaks at -1 : i.e. they tend to be anti-parallel. However, a smaller peak is observed at $\vec{a}_{i} \cdot \vec{a}_{j} \approx 1$. The peak at $\vec{a}_{i} \cdot \vec{a}_{j} \approx-1$ has also been observed in all-atom simulations. The small peak at $\vec{a}_{i} \cdot \vec{a}_{j} \approx 1$ has not been observed in such simulations, and is thus likely an artifact resulting from the absence of methyl groups connected to the steroid rings in our model. If present, these methyl groups would sterically impede this conformation. In general the distributions observed in our simulations are similar to those observed in all-atom simulations for pairs of bile molecules that do not participate in intramicellar hydrogen bonds. These "hydrophobically bonded" molecules make up the majority of the population ${ }^{4}$. The distribution $\vec{a}_{i} \cdot \vec{a}_{j}$ for the small fraction of pairs of bile molecules sharing hydrogen bonds shows peaks that are not seen in our coarse-grained simulations, indicating that hydrogen bonds stabilize certain orientations relative to others. 


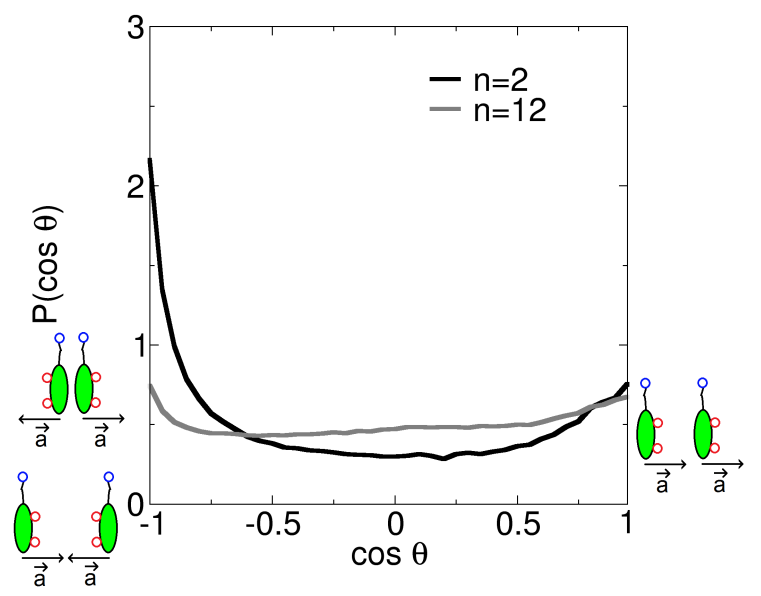

(a)

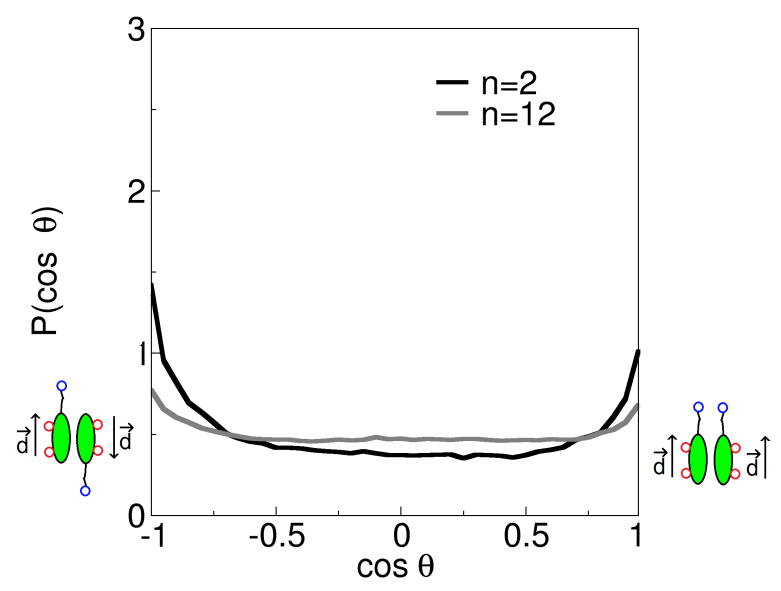

(b)

Figure 11: Distribution of the angles formed by the (a) $\vec{a}$ vectors $\left(\theta=\arccos \left(\vec{a}_{i}\right.\right.$. $\left.\vec{a}_{j}\right)$ ) and (b) $\vec{d}$ vectors $\left(\theta=\arccos \left(\vec{d}_{i} \cdot \vec{d}_{j}\right)\right)$ of neighboring bile salts $(\{\mathrm{ij}\})$ in dimers (black) and in micelles with $\mathrm{n}=12$ (gray) of $2 \mathrm{OH}$ bile salts. The images on either side of each graph illustrate the preferred orientation of neighboring bile salts. 


\section{Concluding remarks}

Our coarse-grained simulations of bile salts reproduce the known average aggregation numbers and micellar shapes of these substances. The model slightly overestimates the critical micelle concentrations, which limits its applicability to predict CMCs of mixed micelles but does not affect the model's usefulness to investigate the stability and structure of bile micelles.

The micelle size distributions obtained here suggest that the free energy barrier for micelle disassembly is comparable to $k_{B} T$ even at concentrations $\approx 8 \times \mathrm{CMC}$. Experiments support this observation ${ }^{21}$. We propose that the high $\mathrm{CMC}$ of bile salts and low micelle stability may be of biological significance. The high CMC implies that a large amount of monomeric bile is available in the upper intestinal tract to rapidly form mixed dietary micelles with the products of enzymatic breakdown of fats. It seems likely that bile solubilizes these breakdown products, yet the micelles are not very stable, thus ensuring that nutrients can easily be released at the intestinal wall. We are currently investigating this possibility by computing the relative stability of mixed micelles as a function of bile salt content.

The size and shape of surfactant aggregates may often be predicted by calculating the molecular packing parameter, $A /(\mathcal{V} \times \ell)$, where $A$ is the effective area of the head group, $\mathcal{V}$ the volume and $\ell$ the length of the hydrocarbon chain. Since our results indicate that the charged group in bile molecules stays preferentially on the outside of the micelle and thus acts as the head group, it is tempting to calculate the packing parameter for bile salts and use it to predict the size and shape of equilibrium aggregates with other surfactants. However, our results suggest that the predictive application of the packing parameter may not work for bile salts as they may pack in many different orientations, both relative to the micelle as a whole and with respect to their neighbors.

We propose that the ability of bile salts to pack in many different orientations in micelles is of biological significance. The low intramicellar order may explain why pure bile salts cannot form (undesirable) smectic phases at physiological concentrations $^{3}$, which may reduce the incidence of these phases in the multicomponent intestinal environment. We hypothesize that low intramicellar order may also facilitate the formation of mixed micelles with nutrients that necessarily come in a wide range of shapes, sizes and hydrophilicity. Since most nutrients resulting from the enzymatic breakdown of fats are poorly soluble and would thus tend to form large aggregates, the bile salt's tendency to form small single component micelles may cap the size of mixed micelles, thus facilitating rapid transport across the $500 \mu \mathrm{m}$-thick polysaccharide layer that covers the intestinal walls.

The coarse-grained model of bile salts presented here retains sufficient detail to provide molecular-scale insight into bile salt aggregation. This model can be used 
in conjunction with existing models of head-tail amphiphiles representative of nutrients in the intestinal tract ${ }^{16}$ to gain further insight into the physical-chemistry of digestion. This model can also easily be modified to simulate other unusual

amphiphiles, such as asphaltenes or bolaamphiphiles ${ }^{30,31}$. The low number of degrees of freedom of these models makes them ideal to investigate local and global equilibrium states and transition paths between them.

\section{Acknowledgments}

The work of the FOM Institute is part of the research program of FOM and is made possible by financial support from the Netherlands organization for Scientific Research (NWO). AV acknowledges support from Senter-Novem and the Dutch Ministry of Economic Affairs through the Food \& Nutrition Delta 2 Program (grant DFN0642300) for a joint FOM-Unilever project. DF acknowledges financial support from the Royal Society of London (Wolfson Merit Award) and from the ERC (Advanced Grant agreement 227758). Drs. Sanne Abeln, Axel Arnold, Aimee Bailey, Rob Groot and Krassimir Velikov are cordially thanked for their helpful comments.

\section{References}

[1] W. H. Elliot, P. Eneroth, S. L. Hsia, D. Kritchevsky, A. Kuksis, J. T. Matschiner, P. P. Nair, R. Ryhage, J. Sjovall and D. Small, The bile acids chemistry, physiology, and metabolism, Plenum Press, New $\overline{\text { York - London, }}$ 1971, vol. 1: chemistry.

[2] I. Bekersky, J. B. Carey, H. Danielsson, A. F. Hofmann, T. F. Kellogg, D. Kritchevsky, L. Lack, H. Mekhjian, T. A. Miettinen, E. H. Mosbach, P. P. Nair, R. H. Palmer, M. P. Tyor and I. M. Weiner, The bile acids chemistry, physiology, and metabolism, Plenum Press, New York - London, 1973, vol. 2: physiology and metabolism.

[3] E. F. Marques, H. Edlund, C. La Mesa and A. Khan, Langmuir, 2000, 16, $5178-5186$.

[4] L. Partay, M. Sega and P. Jedlovszky, Langmuir, 2007, 23, 12322-12328.

[5] L. B. Partay, P. Jedlovszky and M. Sega, Journal of Physical Chemistry B, 2007, 111, 9886-9896. 
[6] D. B. Warren, D. K. Chalmers, K. Hutchison, W. B. Dang and C. W. Pouton, Colloids and Surfaces A-Physicochemical and Engineering Aspects, 2006, 280, 182-193.

[7] S. Mukhopadhyay and U. Maitra, Current Science, 2004, 87, 1666-1683.

[8] Y. Sugita, A. Kitao and Y. Okamoto, Journal of Chemical Physics, 2000, 113, 6042-6051.

[9] H. Fukunishi, O. Watanabe and S. Takada, Journal of Chemical Physics, 2002, 116, 9058-9067.

[10] R. Affentranger, I. Tavernelli and E. E. Di Iorio, Journal of Chemical Theory and Computation, 2006, 2, 217-228.

[11] A. P. Lyubartsev, A. A. Martsinovski, S. V. Shevkunov and P. N. Vorontsovvelyaminov, Journal of Chemical Physics, 1992, 96, 1776-1783.

[12] E. Marinari and G. Parisi, Europhysics Letters, 1992, 19, 451-458.

[13] C. J. Geyer and E. A. Thompson, Journal of the American Statistical Association, 1995, 90, 909-920.

[14] Y. Sugita and Y. Okamoto, Chemical Physics Letters, 1999, 314, 141-151.

[15] D. Frenkel and B. Smit, Understanding molecular simulation - from algorithms to applications, Academic press, London, UK, 2nd edn., 2002, vol. 1.

[16] I. R. Cooke, K. Kremer and M. Deserno, Physical Review E, 2005, 72, 011506.

[17] H. J. Limbach, A. Arnold, B. A. Mann and C. Holm, Comput. Phys. Commun., 2006, 174, 704-727.

[18] W. Humphrey, A. Dalke and K. Schulten, Journal of Molecular Graphics, 1996, 14, 33-38.

[19] D. Myers, Surfactant science and technology, Wiley Interscience, Hoboken, New Jersey, 3rd edn., 2006.

[20] A. Roda, A. F. Hofmann and K. J. Mysels, Journal of Biological Chemistry, 1983, 258, 6362-6370.

[21] H. Kawamura, Y. Murata, T. Yamaguchi, H. Igimi, M. Tanaka, G. Sugihara and J. P. Kratohvil, Journal of Physical Chemistry, 1989, 93, 3321-3326. 
[22] M. C. Carey, J. C. Montet, M. C. Phillips, M. J. Armstrong and N. A. Mazer, Biochemistry, 1981, 20, 3637-3648.

[23] N. Funasaki, R. Ueshiba, S. Hada and S. Neya, Journal of Physical Chemistry, 1994, 98, 11541-11548.

[24] D. M. Small, Adv. Chem. Ser., 1968, 84, 31.

[25] N. A. Mazer, M. C. Carey, R. F. Kwasnick and G. B. Benedek, Biochemistry, 1979, 18, 3064-3075.

[26] R. Pool and P. G. Bolhuis, Journal of Physical Chemistry B, 2005, 109, 6650-6657.

[27] P. G. Bolhuis and D. Frenkel, Physica A, 1997, 244, 45-58.

[28] D. G. Oakenfull and L. R. Fisher, Journal of Physical Chemistry, 1977, 81, 1838-1841.

[29] P. Garidel, A. Hildebrand, R. Neubert and A. Blume, Langmuir, 2000, 16, $5267-5275$.

[30] G. Andreatta, C. C. Gonalves, G. Buffin, N. Bostrom, C. M. Quintella, F. Arteaga-Larios, E. Perez and O. C. Mullins, Energy \& Fuels, 2005, 19, $1282-1289$.

[31] A. H. Fuhrhop and T. Y. Wang, Chemical Reviews, 2004, 104, 2901-2937. 